\title{
Pinpointing Tourism Information onto Mobile Maps - A Light-Weight Approach
}

\author{
W. Schwinger ${ }^{1}$, C. Grün ${ }^{2}$, B. Pröll ${ }^{1}$, W. Retschitzegger ${ }^{1}$, H. Werthner ${ }^{2}$ \\ ${ }^{1}$ Johannes Kepler Universität Linz, Altenbergerstraße 69, A-4040 Linz \\ http://www.uni-linz.ac.at \\ ${ }^{2}$ Leopold-Franzens-Universität Innsbruck, Universitätsstraße 15, A-6020 \\ http://iset.uibk.ac.at
}

\begin{abstract}
Location-based systems in the area of tourism, so-called mobile tourist guides, combine geographic information and tourism information in order to deliver relevant content to tourists on the spot. Existing systems often adhere to a heavy-weight approach. This means that, firstly, some do not build on existing tourism information systems, secondly, most of them do not employ standards for accessing geographical information systems, and thirdly, the majority employ a thick client. To tackle these issues, we propose a lightweight approach for location based systems. A major focus is on configurability, allowing to easily making existing tourism information location-aware, by pinpointing points of interest to geographical positions on a map. To demonstrate the applicability of the framework, a prototype of a mobile tourist guide has been implemented for the city of Linz, Austria, which delivers geographic data in form of maps or aerial photos and information about local points of interest.
\end{abstract}

\section{Motivation}

Location-based systems (LBS) developed in the area of tourism provide so-called mobile tourist guides by delivering relevant information to tourists on the spot, therefore more and more supplementing traditional maps and guidebooks. Thus, a mobile tourist guide combines a geographic information system (GIS) and a tourism information system (TIS) (Baus et al. 2005; Berger et al. 2003).

Geographic data in form of mobile maps represent one cornerstone, since nearly all tourism relevant information link to a geographic location. Such a map provides the tourist with a snapshot of his/her environment at a certain location and time, presenting highly selective information relevant to the tourist, which has to be generated on the fly to be up-to-date (Meng \& Reichenbacher, 2005). Tourists' activities can be enhanced through different kinds of maps, ranging from a street map for city-trips to a topological map used for mountain tours. A single GIS can hardly provide all those kinds of maps. Furthermore, a mobile tourist guide should be employed in different geographic regions, probably supported by alternative GIS. Thus, a mobile tourist guide has to be open to be adjusted to various GIS.

Tourism data, representing the second cornerstone of mobile tourist guides, is crucial in that it is obviously subject to dynamic changes, as attractions can be temporarily closed, restaurants can change their special offers on a weekly basis, and open-air concerts can be cancelled due to bad weather conditions. For realizing a mobile tourist guide, a centralized approach whereby all that data is kept in a single collective repository is certainly not feasible neither in terms of maintenance effort nor since 
there is already a huge amount of tourism data available (Haller et al. 2000). Furthermore, and most important, flexibility should be ensured so that the mobile tourist guide can be deployed also in other tourism regions with little effort.

Several mobile tourist guides have already been developed (Anegg et al. 2002; Cheverst et al. 2002; Garzotto et al. 2004; O'Grady \& O'Hare, 2004; Hinze \& Voisard, 2003; Hofer et al. 2003; Kamar, 2003; Krösche et al. 2004; Poslad et al. 2001; van Setten et al. 2004). However, most of them adhere to a heavy-weight approach. Firstly, most of them do not build on existing tourism information. Secondly, the majority of them do not rely on existing standards to access already existing geographic and tourism data. Thirdly, many of them require extensive computational power on the client side for several reasons, thus limiting the applicability of various mobile devices.

To tackle these shortcomings, this paper introduces a light-weight framework for location-based systems, integrating existing GIS and TIS information (Schwinger et al. 2005). In contrast to most other systems, it is not the main goal to implement a full-fledged mobile tourist guide providing all kinds of tourism and geographic services which could support the mobile tourist, e.g. routing, recommendations, and guided tours. Rather the focus is on high configurability to make existing tourism information location-aware by linking points of interest (PoIs) to geographical positions on a map. To demonstrate its applicability, a prototype of a mobile tourist guide has been implemented for the city of Linz in Austria that delivers geographic data in form of maps or aerial photos and information about PoIs to mobile devices.

The structure of the paper is as follows. Section 2 discusses the design goals and design alternatives pursued in the development of our framework. Based on that, Section 3 gives an overview of the functionality of the framework, whereas Section 4 discusses the underlying architecture and its implementation. The benefits of our approach are discussed with respect to closely related approaches in Section 5. Finally, Section 6 puts forward the experiences we gained from our prototype in form of lessons learned.

\section{Design Alternatives for Combining GIS and TIS}

This section discusses design alternatives for integrating GIS and TIS, together with design goals and corresponding design decisions taken for our light-weight framework of a location-based system. The design alternatives can be grouped along three dimensions, comprising geographic capabilities and tourism capabilities which both provide the main constituents of a mobile tourist guide as well as configuration capabilities with respect to these two dimensions (Fig. 1). It has to be mentioned that the design alternatives represent only a subset of all possibilities which we consider to be relevant for developing our framework, nevertheless providing a proper basis for developing similar systems. Note that some of the design alternatives represent complementary possibilities whereas others are mutual exclusive and some categories of design alternatives are not exhaustive by nature.

Since our overall goal is to provide a light-weight framework, the design decisions have to consider certain technical prerequisites influencing all of the above mentioned dimensions. First, a light-weight approach should support a broad range of mobile devices thus involving only minimal computational power at the client side, i.e., a thin 
client approach. Second, since caching techniques can not be employed with a thin client, a permanent network connection is needed to retrieve data form the server. Third, and most important, minimal effort shall be necessary to realize mobile tourist guides combining GIS and TIS data.

\subsection{Design Alternatives with Respect to Geographic Capabilities}

Geographic Services. Besides the elementary service of positioning, geocoding services allow to determine $\mathrm{x} / \mathrm{y}$-coordinates for PoIs, map services provide basic map functions such as zooming or panning which can be enhanced by routing services computing routes between certain locations. Geographic searches offer the possibility to find certain PoIs; optionally in certain distance to the user then called proximity searches. Our design goal is to support a core set of such geo-services.

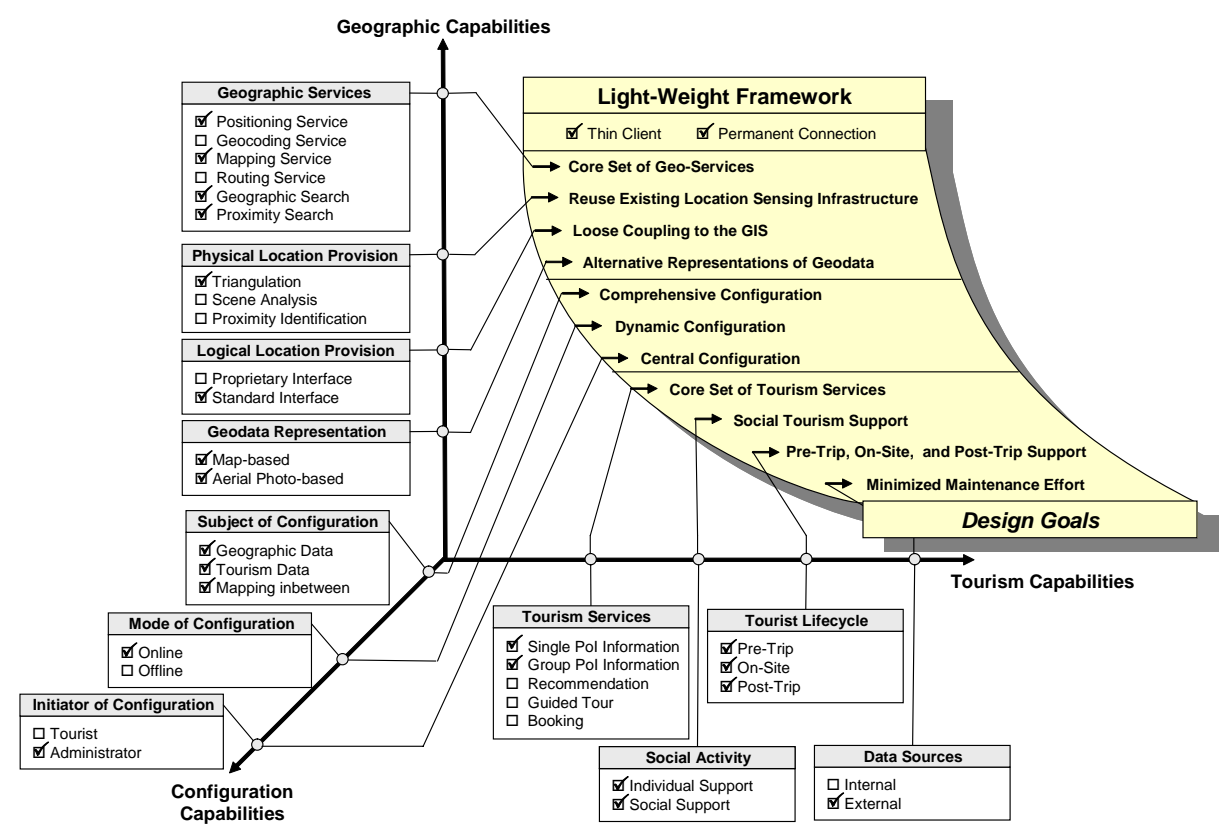

Fig. 1. Design Alternatives and Design Goals

Physical Geodata Provision. Three main technologies of physical geodata provision, i.e. the provision of the current location can be distinguished, comprising triangulation (e.g. GPS), scene analysis (e.g. observing shape of objects in the scene) and proximity identification (e.g. RFID tags attached to physical locations) (Hightower \& Borriello, 2001). An important design goal is the usage of existing location sensing infrastructure. Since proximity identification needs additional infrastructure and scene analysis requires high computational power, thus not in line with our overall goal of a light-weight approach, we rely on GPS for physical location provision.

Logical Geodata Provision. Logical geodata (e.g. maps) complementing the physical geodata can be provided either through a proprietary interface or using a standard, 
e.g. the Open Location Services, defined by the Open Geospatial Consortium (OGC). Since another design goal is to achieve loose coupling to the GIS in order to enable a rapid deployment of the system in geographic regions covered by other GIS, a standard to query and exchange geodata is of importance.

Geodata Representation. Geodata can be represented in form of various kinds of maps, e.g. street maps or aerial photos. The goal of our framework is to support alternative representations of the geodata to provide the user with roads, buildings and other infrastructure information as well as aerial photos for a general impression of the geography.

\subsection{Design Alternatives with Respect to Tourism Capabilities}

Tourism Services. Besides the basic functionality of providing tourists with information about single PoIs and groups of PoIs according to common criteria (e.g. all restaurants), mobile tourist guides could actively recommend PoIs or even guided tours to the tourist based on personal preferences and could in addition offer booking services. Since our design goal is to offer a basic set of tourism services with minimal implementation effort, the framework focuses on single PoIs and groups of PoIs only. Social Activity. Tourists tend to explore an attraction, not only individually but together with others, so to share the tourism experience in a collaborative way. In our framework we pursued the design goal of supporting also the social aspect of tourism by adding core collaborative features.

Tourist Life Cycle. The main focus of mobile tourist guides is on providing tourists with up-to-date information during their trip, but also pre-trip and post-trip support would be imaginable. Our design goal is to consider, beside the on-site support which is the major focus, also basic support for pre-trip and post-trip phase.

Data Sources. Tourism data can be maintained internally by the mobile tourist guide or retrieved from external sources, e.g. existing TIS. Since one of our design goals is to minimize maintenance effort, our framework bases on already existing tourism data.

\subsection{Design Alternatives with Respect to Configuration Capabilities}

Subject of Configuration. Mobile guides can offer configuration capabilities, on the one hand with respect to the GIS (e.g. access parameter settings) as well as the TIS (e.g. PoIs provided) and on the other hand with respect to the linkage of geodata to tourism data. The design goal is to allow for comprehensive configuration (Kapsammer et. al 2005), to incorporate various geographic and tourism data, thus supporting all three types of configuration subjects.

Mode of Configuration. Regarding the mode of configuration one can distinguish between an offline mode (e.g. by means of a XML configuration file) or an online mode (e.g. through a web-interface). To allow for dynamic configuration representing another design goal of our framework, we adhere to the online mode.

Initiator of Configuration. The necessary configuration can either be performed by each individual user before the trip, which provides for high configurability but, as a drawback, requires a lot of configuration effort. Or the configuration can be conducted by an administrator of the system which liberates the user of this burden and will allow for a central quality control. To achieve the design goal of central configuration, an administrator will be responsible for the general configuration. 


\section{Functionality of the Framework}

Based on the design goals identified in the previous section a brief overview of the basic features of the framework is given in the following, structured into geographic capabilities, tourism capabilities, and configuration capabilities. Basically the framework provides tourists, equipped with a mobile device such as a PocketPC (Fig. 2), with a map and PoI information tailored to their current location and interests (Kappel et al. 2003). PoIs are marked on the map through symbols to visualize their spatial relationship. By pointing on them, the user gets detailed information about the selected site. The framework is based on a Web browser as client, a GPS sensor for physical location provision and a Web Server. For details about the implementation it is referred to Section 4.

\subsection{Geographic Capabilities}

Visualization of Current Position. The user's position is visualized on the map by a circle as symbol to indicate the position's uncertainty. Even if the GPS device can calculate a valid position, the real user's position can differ up to 5 meters because of disturbances in the atmosphere (Hightower \& Borriello, 2001).

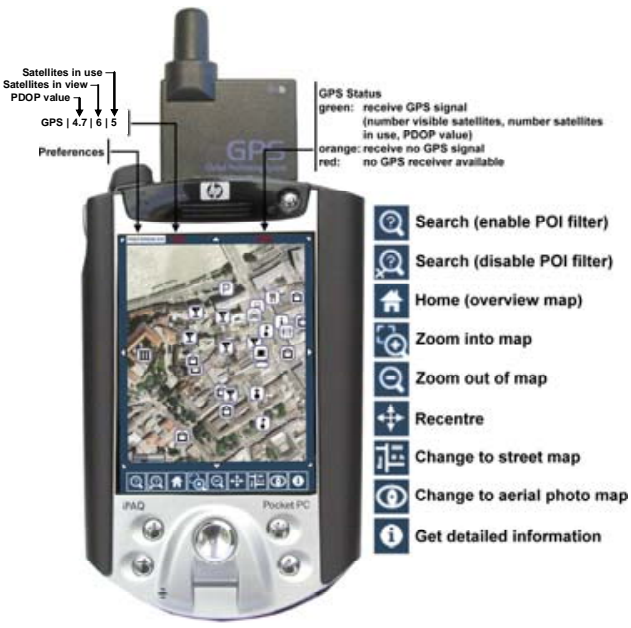

Fig. 2. Map Screen Layout

Refreshing of Position. The displayed position together with the map is refreshed automatically either as soon as the user performs an explicit map action, e.g. panning, or periodically to reflect the movement of the user. The user is able to adjust the refreshing period to his/her speed of movement. The user has also the possibility to completely turn off this feature.

Basic Map Functionality. The framework supports basic map functionalities like panning, selecting a PoI symbol or changing the zoom level. In addition, the user can switch between a street map and aerial photos.

Proximity Search. The user is able to search PoIs indicating a certain distance as search radius. If the user's location cannot be determined, the center of the currently displayed map is assumed to be the current user's position. 
Indication of Position Accuracy. The unavailability and changing accuracy of the GPS signal is dealt with by continuously informing the user about its quality. The number of satellites visible, those which are used to fix the position and the positional dilution of precision (PDOP) value are measured and displayed in the upper right corner of the screen. The PDOP value indicates the quality of the geometric orientation of the satellites being used to compute the position.

Map Screen Layout. The map screen (Fig. 2) is composed of three elements. At the bottom, functional buttons (in form of Soft Keys) provide general application control options. In the center, an interactive map is shown, allowing the user to invoke further functionality by clicking on a certain position (e.g. choosing a PoI symbol). At the top, information about the physical location's availability is presented as well as a "Preferences" button that allows to configure additional settings.

\subsection{Tourism Capabilities}

Information about PoIs. PoIs are marked on the map through self-explaining, pictogram-like symbols (Fig. 3). If a PoI contains more information, its symbol is displayed with full opacity, indicating that it is clickable, showing, e.g. historic information, opening hours or pictures, content which is provided by the existing TIS TIScover (Pröll \& Retschitzegger, 2000; TIScover).
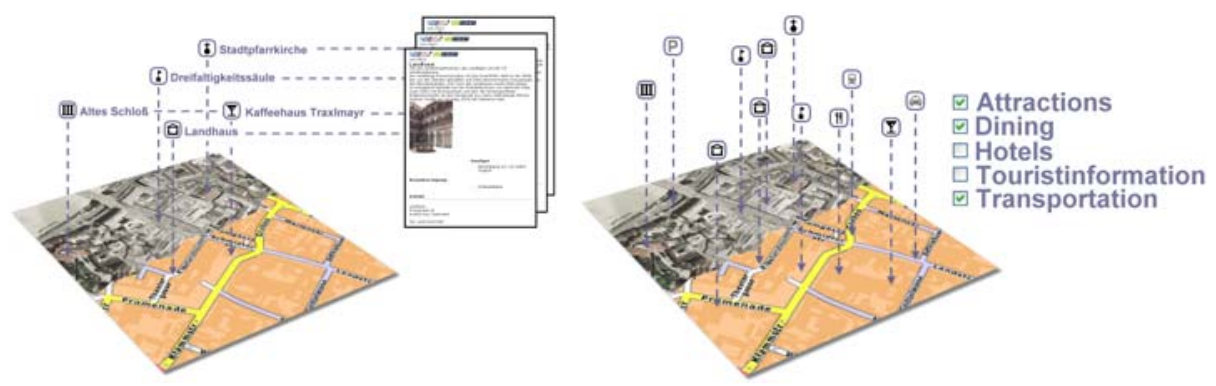

Fig. 3. PoIs and Thematic Layers

Selection of Thematic Overlays. PoIs are categorized into, possibly nested, thematic layers, e.g. attraction or dining, in order to avoid the small screen being cluttered (Fig. 3 ). The set of PoIs, chosen by the user, including those PoIs associated with subcategories, is then superimposed on the map at each user request.

Support of Social Activity. Basic collaboration functionality is provided in two ways. Firstly, PoIs provide a link to a guestbook (e.g. GeekBlog) allowing users to annotate PoIs (e.g. rating the quality of a restaurant), that can be shared with other users through a so called guest book screen (Fig. 4). Secondly, the system offers the visualization of other users simultaneously using the system. The positions of registered friends are marked on the map, thus providing the prerequisite for subsequent interaction.

Support of Tourist Life Cycle. Besides on-site support, the framework offers also a basic kind of pre-trip support in that users can browse for information by explicitly entering a fictitious position. Post-trip, tourists can revisit their trips in that they are enabled to annotate the visited PoIs which is also available after a tour. 
Information Screen Layout. The screen presenting detailed tourism information is split up into two frames. The top frame consists of a small menu, providing links to TIScover, the guestbook screen, and back to the map screen.

The three screens supported by our framework are together depicted in Fig. 4: One for geographic data (map screen), one for the tourism data (information screen), and one for the annotated PoIs (guestbook screen). The left screenshot shows the street map of the city center of Linz with some PoI symbols. When a PoI is selected (e.g. the PoI "Dreifaltigkeitssäule", displayed on the map of the left screenshot through the flagsymbol), the information screen is loaded and presents detailed information. The screenshot on the right demonstrates the facility of annotating PoIs with comments.

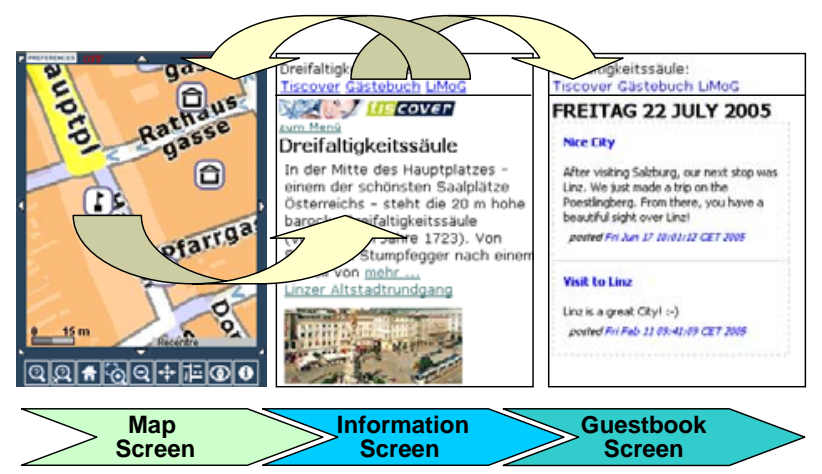

Fig. 4. Different Screens Supported by our Framework

\subsection{Configuration Capabilities}

For configuration of the connection to the existing GIS and TIS information as well as the mapping in-between, the framework provides an easy-to use web-interface. In this way, the administrator can include maps from different GIS as well as manage the different PoIs and the linkage of them to web pages containing more information.

Configuration of External GIS Inclusion. The WMS (Web Map Service) request to the external GIS server can be configured through a Web-based interface. This comprises the URL of the GIS server, the type of map (e.g. aerial photo), which area to retrieve, the spatial reference system (e.g. Gauss-Krüger), the desired output format (i.e. JPEG, PNG or SVG) and the output size of the map in pixels.

Configuration of External TIS Inclusion. In addition to configuring the integration of external GIS content, the framework offers the possibility of configuring the inclusion of external tourism data similar to (Krösche \& Boll, 2005), through a Webbased interface (Fig. 5). Firstly, PoIs can be added, deleted and updated in the framework's repository (cf. Section 4). Secondly, for each PoI a title, the location in form of geographical coordinates and an URL pointing to external tourism data describing the PoI in more detail, can be configured. Thirdly, PoIs can be assigned to possibly nested categories, thus forming thematic layers such as attractions, including the sub categories of buildings, churches and museums. Fourthly, for each category the visualization of the corresponding PoIs can be chosen in terms of a pictogram. 


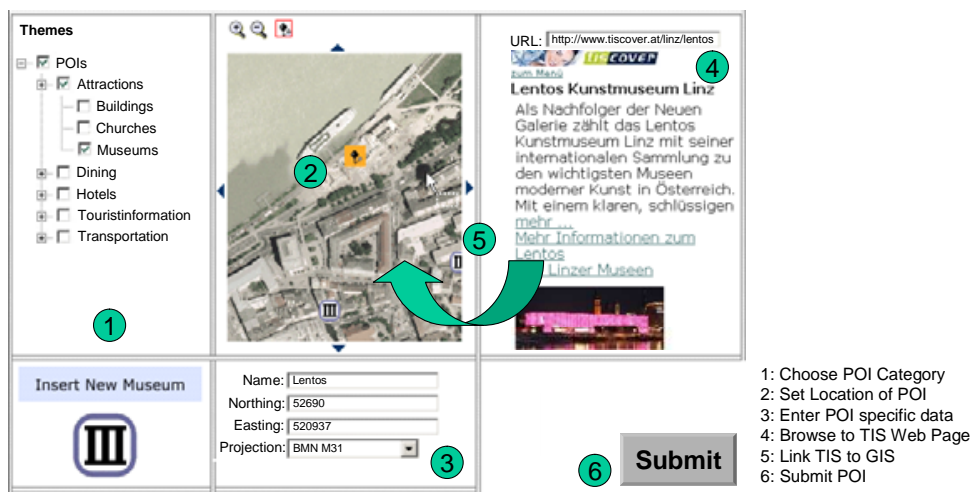

Fig. 5. Web-Interface for GIS-TIS Mapping (Adding a New PoI)

\section{Architecture and Implementation}

The architecture of our framework is based on a client/server approach. It comprises internal components in terms of a Client, a LBS Engine and a Repository (Fig. 6). External components comprise a GIS Server and an external Web Servers in terms of a TIS to deliver further information about PoIs.

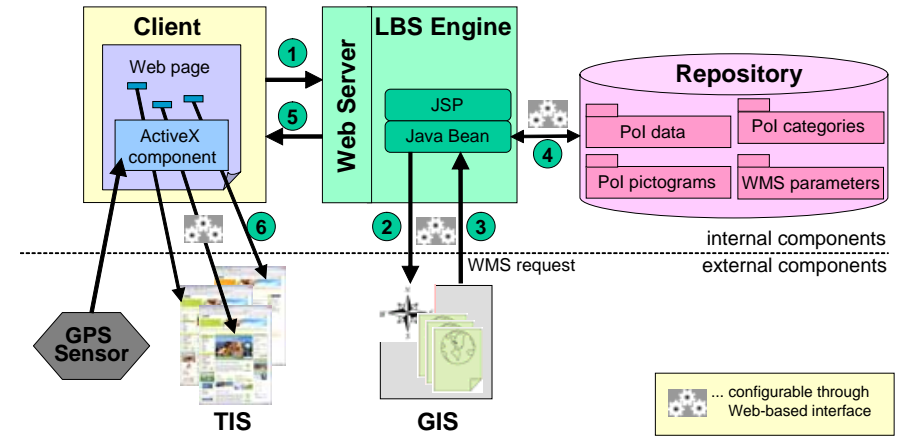

Fig. 6. Architecture of our Framework

The Client. Every mobile device with wireless connectivity, a Web Browser capable of using ActiveX controls and a GPS sensor can serve as a client. Apart from obtaining GPS coordinates from the GPS sensor, all application logic resides at the LBS Engine on the Web Server. This way a thin client with little memory and CPU power is capable of running the Web application.

The LBS Engine. The LBS Engine on the Web Server is the core of the framework and is implemented using JSP and Java Beans. It accepts requests from the client as input and queries the appropriate content. Based on the user's location and preferences it gathers geographic and tourism data from different data sources. Each time the user carries out an action concerning the map presentation, a new map with the appropriate scale is requested from the GIS server. The LBS Engine then accesses the Repository to retrieve the PoI's configuration. All PoIs that belong to the visible 
section of the map and to the categories selected by the user are displayed on the map. The size of the PoI symbols is adjusted according to the selected zoom level. A transformation has to be applied to the PoI coordinates. The map augmented with the PoIs is then transferred to the client in the form of a MIME-type encoded picture.

The Repository. The Repository holds all information about the PoIs and parameters necessary for configuring a WMS request. The Repository uses HSQL (Hypersonic SQL database), a small, open-source database written in Java. All PoI data and WMSrelated parameters can be managed over a Web-based interface.

Processing a Request. On each request the system performs the following steps:

(1) The Web Browser communicates with the Web Server over WLAN and is continuously informed about the user's location. Position readings from the GPS receiver are sent every two seconds via the COM-Port of the Pocket PC to an ActiveX control element, embedded in the corresponding HTML-page, that parses them. As the user invokes actions, e.g. clicking on a PoI, a request with the GPS data is sent to the Web Server and forwarded to the LBS Engine for processing.

(2) and (3): Corresponding to the user's position and kind of request, the LBS Engine requests geodata from the GIS Server using the WMS standard of the OGC. A WMS allows spatial data to be shared via HTTP in the form of maps which can be raster images in JPEG, GIF, PNG or SVG format. As a prerequisite, the LBS Engine requests the configured WMS parameters from the Repository.

(4) Before being deployed to the Web Browser, the map image is, based on the user's preferences and location, augmented with information about PoIs retrieved from the Repository. The PoIs' geographical coordinates are transformed into pictures' pixel coordinates to correctly locate the PoI pictograms on-top of the map.

(5) The user receives the image, which contains the map and thematic layers.

(6) When the user requests further information of a specific PoI, the corresponding Web page is retrieved from the appropriate external Web Server.

\section{Related Work}

Recently, a proliferation of approaches for mobile tourist guides can be observed (Anegg et al. 2002; Cheverst et al. 2002; Garzotto et al. 2004; O'Grady \& O'Hare, 2004; Hinze \& Voisard, 2003; Hofer et al. 2003; Kamar, 2003; Krösche et al. 2004; Poslad et al. 2001; van Setten et al. 2004). This section gives an overview of selected approaches most related to our light-weight framework approach. For an in-depth comparison of different approaches the reader is referred to (Schwinger et al. 2005).

COMPASS (van Setten et al. 2004) - short for COntext-aware Mobile Personal ASSistant - supports tourists with context-aware recommendations and services. COMPASS makes use of existing geographic and tourism information, but differently to our approach, by using web services. It uses a proprietary interface for getting geographical data. Configuration is done automatically in that those web services that do not match certain hard-coded criteria are filtered out. Mapping between GIS and TIS content is limited in that defining new PoIs at specific locations on the map is not possible, since they are provided through an external web service.

LoL@ (Anegg et al. 2002) - short for Local Location Assistant - is a research prototype of a location-based mobile application for GPRS/UMTS, providing tourists 
with multimedia tourism information about the city of Vienna. Opposite to our approach, all tourism information resides in an own repository, therefore requiring maintenance effort. GIS information is obtained through an external map service, but without using a standard interface. LoL@ does not seem to provide a configuration mechanism of inter-linking GIS and TIS information in a dynamic manner.

Gulliver's Genie (O'Grady \& O'Hare, 2004) is a prototype stemming from the area of artificial intelligence and agent systems focusing on intelligent content delivery to tourists in a proactive manner. Gulliver's Genie presents tourists a map with their current position and personalized multi-media content about cultural attractions. In this respect Gulliver's Genie has a somewhat different focus than our approach since it emphasizes on the presentation of tourism information rather than on the reuse of existing geographic and tourism data, which is in fact managed in a DB2 database.

MobiDENK (Krösche et al. 2004) - German acronym for Mobile Monuments provides tourists with information about monuments. It presents tourists a map of their environment, which is dynamically loaded from a GIS server through an OGC WMS. However, tourism content is only provided through an internal database. Online configuration of the mapping between GIS and TIS seems not foreseen.

The $m$-ToGuide prototype (Kamar, 2003) has been developed to promote the use of 2.5/3G cellular networks with LBS and offers tourists information about PoIs. It is not stated how geospatial data is accessed. XML technology is employed to integrate content from different providers; configuration is done in an offline manner only.

Fig. 7 summarizes the comparison by giving an overview on the system's access mechanisms to TIS and GIS as well as the configuration capabilities and the technical prerequisites in terms of thick and thin clients.

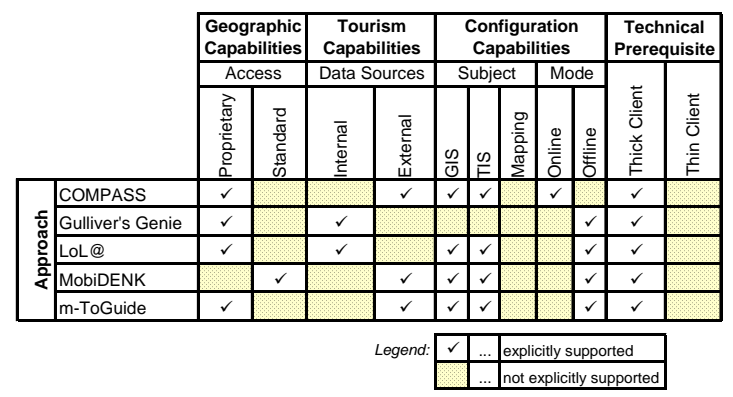

Fig. 7. Summary of the Comparison

Existing approaches often use proprietary interfaces to access a GIS, thus falling short in portability. Some approaches employ their own data repositories, leading to high maintenance costs. Existing solutions are sometimes limited, especially concerning configuration capabilities between GIS and TIS as well as the support of an online configuration mode. It has to be noted that all of the compared approaches use thick clients and are therefore constrained in the usage of various mobile devices.

\section{Concluding Lessons Learned}

The applicability of our framework has been demonstrated by realizing a mobile tourist guide for the city of Linz called LiMoG - short for Linzer Mobile Guide. The 
configured PoIs include e.g. historic sights, churches and cafés along sightseeing tours through the historic centre of Linz in Austria. DORIS (DORIS), the GIS of the federal state of Upper Austria, provides the geospatial data in form of an OGC WMS. Each PoI links to an existing Web page provided by Austria's official destination information and booking system TIScover (Pröll \& Retschitzegger, 2000; TIScover). For demonstration purposes, we used a Pocket PC equipped with a GPS receiver. For accessing the Internet, a publicly available WLAN hotspot was utilized.

Emerging from our experiences we gained from developing the framework and testing the operability of LiMoG, the following lessons learned were identified. They can be split up into lessons learned concerning the configuration and representation of PoIs, the supported phases of the tourist life cycle, and the technology used.

Decentralized Configuration of PoIs Desired. The configuration of the PoI repository should not only follow a central approach in that the administrator (e.g. a local tourist office) alone can add new PoIs. A community managing its own PoIs and being aware of its own tourism knowledge can be established if the capability to configure and add new data is granted to other stakeholders.

Semi-Automatic Configuration of PoIs Needed. Setting up a PoI repository is demanding, especially when the tourist guide supports tourists in a large region. To lower this effort PoIs and categorisations thereof could be semi-automatically extracted from a TIS, as a TIS already maintains a large amount of PoIs. Prerequisite is that the geographical location of each PoI is available in the TIS database.

Resolving Overlapping PoI Symbols Necessary. The switching between thematic layers, to reduce the number of PoIs displayed, does not always prevent the overlapping of PoI symbols on a map, especially in areas with a high density of PoIs. Concepts of reconfiguring the arrangement of PoI symbols to resolve overlapping but at the same time preserve spatial relationship have to be applied.

Aerial Representation of PoIs Useful. The concept that a PoI is assigned to a single pair of $\mathrm{x} / \mathrm{y}$-coordinates should be extended to represent whole areas relevant to the tourist, e.g. a square, in terms of polygons. This is also particularly relevant since tourists often seek a specific tourist area, e.g. Chinese quarter, to find a particular sort of tourist attraction (e.g. a Chinese restaurant).

Support of Post-Trip Phase Wanted. When tourists return from a tour, they like to have a reminder of the trip and share their travel experiences. With respect to this, we encountered similar to (Brown \& Laurier, 2005), that besides simple annotations also automatic tracing of the tour and composition of tour summaries are desired.

Usage of Hybrid Positioning Methods Beneficial. GPS can cause problems due to alleyways blocking the signals and should not be the sole location sensing technology used. Enhance accuracy can be gained by using a technology such as Assisted GPS (A-GPS), combining cellular positioning methods with GPS (Swedberg, 1999). Another concept to assist positioning is Active Landmarks, using prominent PoIs as reference points for location provision (Gartner \& Uhlirz, 2005).

Coverage of Broad Spectrum of Network Technologies Required. In mobile scenarios the device is especially exposed to network fluctuations or even loss of network connection. When pursuing a thin client approach, caching is not an option, thus requiring a set of network technologies which is able to deal with this problem. This can be for example WLAN hotspots in the urban areas, whereas GPRS/UMTS might be the only available technology that can be used in rural areas. 


\section{References}

Anegg, H. et al. (2002). Lol@: Designing a Location Based UMTS Application. ÖVE-Verbandszeitschrift, Ausgabe 02/2002 e\&i, Springer.

Baus, J. et al. (2005). A Survey of Map-based Mobile Guides. In Liqiu Meng and Alexander Zipf (eds.): "Map-based mobile services - Theories, Methods and Implementations". Springer.

Berger, S., Lehmann, H., Lehner, F. (2003). Location-based Services in the tourist industry. International Journal on Information Technology \& Tourism, Cognizant, 5(4).

Brown, B., Laurier, E. (2005). Designing electronic maps: an ethnographic approach. In A. Zipf, L. Meng, T. Reichenbacher: Map-based mobile services - Theories, Methods and Implementations. Springer.

Cheverst, K. et al. (2002). Developing a context-aware electronic tourist guide: some issues and experiences. Proc. of the SIGCHI Conference on Human Factors in Comp. Systems, The Netherlands.

DORIS, Digitales Oberösterreichisches Raum-Informations-System. http://doris.ooe.gv.at.

Gartner, G., Uhlirz, S. (2005). Cartographic Location Based Services. In A. Zipf, L. Meng, T. Reichenbacher (eds) Map-based mobile services - Theories, Methods and Implementations. Springer.

Garzotto, F. et al. (2004). Ubiquitous Access to Cultural Tourism Portals. Proc. of the Int. Workshop on Presenting and Exploring Heritage on the Web (PEH). Spain.

GeekBlog. http://geekblog.sourceforge.net.

Haller, M., Pröll, B., Retschitzegger, W., Tjoa A. M., Wagner, R. (2000). Integrating Heterogeneous Tourism Information in TIScover - The MIRO-Web Approach. Information and Communication Technologies in Tourism, ENTER 2000, Barcelona.

Hightower, J., Borriello, G. (2001). A Survey and Taxonomy of Location Systems for Ubiquituous Computing. University of Washington, Computer Science and Engineering

Hinze, A., Voisard, A. (2003). Location- and Time-based Information Delivery in Tourism. Proc. of the 8th Symposium on spatio-temporal databases.

Hofer, T., Pichler, M., Leonhartsberger, G., Schwinger, W., Altmann, J., Retschitzegger, W. (2003). Context-Awareness on Mobile Devices - the Hydrogen Approach. Proc. of the 36th HICSS, Hawaii.

HSQL. Database Enginge. http://hsqldb.org.

Kamar, A. (2003). Mobile Tourist Guide (m-ToGuide). Deliverable 1.4, Final Report.

Kappel, G., Pröll, B., Retschitzegger, W. Schwinger, W. (2003). Customisation for Ubiquitous Web Applications - A Comparison of Approaches. Int. Journal of Web Engineering and Technology.

Kapsammer, E. Retschitzegger, W. Schwinger, W. (2005). Bridging Relational Databases to ContextAware Services. Proc. of the CAiSE Workshop UMICS, Portugal.

Krösche, J., Baldzer, J., Boll, S. (2004). MobiDENK - Mobile Multimedia in Monument Conservation Conservation. In: IEEE MultiMedia, 11(2), IEEE, S.72-77.

Krösche, J., Boll, S. (2005). The xPoI Concept. In: International Workshop on Location- and ContextAwareness (LoCA 2005) in conjunction with Pervasive 2005, Germany.

Meng, L., Reichenbacher, T. (2005). Map-based mobile services. In A. Zipf, L. Meng, T. Reichenbacher (eds) Map-based mobile services - Theories, Methods and Implementations. Springer.

OGC. Open Geospatial Consortium. http://www.opengeospatial.org.

O'Grady, M., O'Hare, G. (2004). Gulliver's Genie: Agency, Mobility \& Adaptivity. Computers \& Graphics, 28 (4)

Poslad, S. et al. (2001). CRUMPET: Creation of User-Friendly Mobile Services Personalised for Tourism. In: 2nd Int. Conf. on 3G Mobile Communication Technologies. UK.

Pröll, B., Retschitzegger, W. (2000). Discovering Next-Generation Tourism Information Systems - A Tour on TIScover. Journal of Travel Research.

van Setten, M., Pokraev, S., Koolwaaij J. (2004). Context-Aware Recommendations in the Mobile Tourist Application COMPASS. In Nejdl, W. \& De Bra, P. (Eds.). AH 2004, LNCS 3137, Springer.

Swedberg, G. (1999). Ericsson's mobile location solution. Ericsson Review(4).

Schwinger, W., Grün, C., Pröll, B., Retschitzegger, W. (2005). A light-weight Framework for Locationbased Services, Workshop on Context-Aware Mobile Systems (CAMS). Cyprus.

Schwinger, W., Grün, C., Pröll, B., Retschitzegger, W. (2005). Context-Awareness in Mobile Tourism Guides - A Comprehensive Survey. Technical Report, Johannes Kepler University Linz, IFS/TK. [ftp://ftp.ifs.uni-linz.ac.at/pub/publications/2005/0405.pdf].

TIScover, Destination Management System, http://www.tiscover.com. 